\title{
Stability and chaos with mathematical control of 4-d dynamical system
}

\author{
Maysoon M. Aziz, Dalya M. Merie \\ Department of Mathematics, College of Computer Science and Mathematics, University of Mosul, Iraq
}

\begin{tabular}{l}
\hline Article Info \\
\hline Article history: \\
Received Dec 12, 2019 \\
Revised Apr 25, 2020 \\
Accepted Jun 14, 2020 \\
\hline Keywords: \\
Adaptive control \\
Four-dimensional system \\
Lapiynuov exponent \\
Stabilization \\
Synchronization
\end{tabular}

\begin{abstract}
A new four-dimensional continuous-time system is dealt in this paper. The system employs eight simple terms involving nonlinear terms. The fundamental characteristics of the system are analyzed by means of its equilibrium points, dissipativity, wave form analysis, stability analysis, Lapiynuov Exponents and Kaplan-Yorke dimension. The maximum value of Lapiynuov exponent is obtain as (1.660748) and Kaplan-Yorke dimension obtain as (3.143433471), that show the system is unstable and highly chaotic. As well, an optimal controller by adaptive control strategy is established to be system trajectories are stable. Finally, adaptive synchronization of new system is clarified. Tables are made to compare the graphical and theoretical results of the new system in two cases before and after control.
\end{abstract}

Copyright $(5) 2020$ Institute of Advanced Engineering and Science. All rights reserved.

\section{Corresponding Author:}

Maysoon M.Aziz,

Department of Mathematics,

University of Mosul, Mosul, Iraq.

Email: aziz_maysoon@yahoo.com,aziz_maysoon@uomosul.edu.iq

\section{INTRODUCTION}

In decay years, research on chaotic phenomena has increased dramatically due to the increasing limits of chaotic applications in science and engineering systems [1]. The phenomenon of chaos is caused by sensitivity of opponents to perturbation structural parameters and initial conditions of a few categories of dynamic systems [2-5]. Chaotic signals characterize by random-like nature, broadband spectrum and are aperiodic [6,7]. The conditions of the chaotic system that should be satisfied; First, sensitivity to disturbances in its initial conditions which should result in unpredictability behavior on long time; second, it's not a transitional topology; and third, in phase space the chaotic orbits should be dense [8-9]. Among some of the attractors of chaos evolved in the researches are the, Chen's [10], 4-wing attractor [11], SundarapandianPehlivan [12], Rabinovich system [13-15]. A fundamental properties of chaotic system it possess at least one Lapiynuov exponent greeater than zero. A system that has more than one positive lapiynuov exponents is highly chaotic and becomes extremely sensitive to tiny disturbances in the dynamics of his system [16-17]. Because of the controlability and synchronizability chaos control becomes widespread attention from researches is an indication of benefit in completely different designs such as secure communications, artificial intelligence, and biometric identification [18-20]. One of the principle of lapiynuov stability can effectively settled the dissipative systems [21-24].

This paper consists of seven sections: In Section 2, we presented the new system; it is mainly consist of eight simple terms include two nonlinear terms. In Section 3, we investigated basic characteristics of the new system by means that of equilibrium points, stability, dissipativity, Lapiynuov exponents, Kaplan-Yorke dimension and diagrams. In Section 4, we infer the results of adaptive control of the highly chaotic system with unknown parameter. In Section 5, we made tables to compare the system before control \& after control. In section 6, the concept of Adaptive synchronization technique and its results of the identical chaotic systems with an unknown parameter. In Section 7, we presented the conclusions. 


\section{SYSTEM DESCRIPTION}

The new four-dimensional system has the following equations:

$$
\begin{aligned}
& \dot{x}_{1}=\rho\left(x_{2}-x_{1}\right) \\
& \dot{x}_{2}=a x_{1}-\delta x_{1} x_{3}+x_{4} \\
& \dot{x}_{3}=\varphi x_{1} x_{2}-x_{3} \\
& \dot{x}_{4}=-k x_{1}
\end{aligned}
$$

$x_{1}, x_{2}, x_{3}, x_{4}$ are state variables and $\rho, a, \delta, \varphi, k$ are constants.

$$
\text { Where } a=296.5, \rho=10, \varphi=10, \delta=40, k=8
$$

\section{SYSTEM ANALYSIS}

To analyze a dynamical system first we have to look at its equilibrium points by setting (1) equal to zero, this results only one equilibrium point and it is the origin point $O=(0,0,0,0)$.

\subsection{Stability analysis}

\subsubsection{Characteristic equation roots}

The system to be stable it is necessary and sufficient condition that the eigenvalues of its characteristic equation have negative real parts. The Jacobian matrix of new system $(1)$ through $E=(0,0,0,0)$ is given as:

$$
J=\left[\begin{array}{cccc}
-10 & 10 & 0 & 0 \\
296.5 & 0 & 0 & 1 \\
0 & 0 & -1 & 0 \\
-8 & 0 & 0 & 0
\end{array}\right]
$$

The characteristic equation is:

$$
\lambda^{4}+11 \lambda^{3}-2955 \lambda^{2}-2885 \lambda+80=0
$$

The roots of (3) are:

$$
\begin{aligned}
& \lambda_{1}=-1 \\
& \lambda_{2}=-59.693 \\
& \lambda_{3}=0.026984 \\
& \lambda_{4}=49.666
\end{aligned}
$$

Therefore, the new system is unstable.

\subsubsection{Routh stability criterion:}

The Routh stability test states that the system is stable (all poles in OLHP (Open Loop Half plane)) if and only if all the elements in the first column of the Routh array are strictly positive. In addition the number of poles not in the OLHP is equal to the number of sign changes in the first column [25]. Table 1 refer to Routh stability test.

$$
\begin{aligned}
& a_{4}=1 \\
& a_{3}=11 \\
& a_{2}=-2955 \\
& a_{1}=-2885 \\
& a_{0}=80 \\
& b_{2}=a_{2}-\frac{a_{4} a_{1}}{a_{3}}=-2692.727 \\
& b_{0}=a_{0}-\frac{a_{4}(0)}{a_{3}}=80 \\
& c_{1}=a_{1}-\frac{a_{3} b_{0}}{b_{2}}=-2884.67
\end{aligned}
$$


Table 1. Routh array table of system (1)

\begin{tabular}{l|ccc}
\hline$\lambda^{4}$ & 1 & -2955 & 80 \\
$\lambda^{3}$ & 11 & -2885 & 0 \\
$\lambda^{2}$ & -2692.727 & 80 & 0 \\
$\lambda^{1}$ & -2884.67 & 0 & 0 \\
$\lambda^{0}$ & 80 & 0 & 0 \\
\hline
\end{tabular}

System (1) is unstable, since there are two elements in the first column of Table 1 are less than zero.

\subsubsection{Lapiynuov function}

Assume that the Lapiynuov function of system (1) is:

$$
\begin{aligned}
& V\left(x_{1}, x_{2}, x_{3}, x_{4}\right)=\frac{1}{2}\left(x_{1}{ }^{2}+x_{2}{ }^{2}+x_{3}{ }^{2}+x_{4}{ }^{2}\right) \\
& \dot{V}\left(x_{1}, x_{2}, x_{3}, x_{4}\right)=x_{1} \dot{x_{1}}+x_{2} \dot{x_{2}}+x_{3} \dot{x_{3}}+x_{4} \dot{x_{4}} \\
& \dot{V}\left(x_{1}, x_{2}, x_{3}, x_{4}\right)=306.5 x_{1} x_{2}-30 x_{1} x_{2} x_{3}-10 x_{1}^{2}-x_{3}^{2}+x_{4}\left(x_{2}-8 x_{1}\right)
\end{aligned}
$$

Since $\dot{V}\left(x_{1}, x_{2}, x_{3}, x_{4}\right)>0$, hence new system (1) is unstable.

\subsection{Dissipativity}

$$
\text { Let, } f_{1}=\frac{d x_{1}}{d t}, f_{2}=\frac{d x_{2}}{d t}, f_{3}=\frac{d x_{3}}{d t} \text { and } f_{4}=\frac{d x_{4}}{d t} \text {. }
$$

The vector field $\boldsymbol{V}$ that we get:

$$
\left(\dot{x_{1}}, \dot{x_{2}}, \dot{x_{3}}, \dot{x}_{4}\right)^{T}=\left(f_{1}, f_{2}, f_{3}, f_{4}\right)^{T}
$$

Then the divergence of $\boldsymbol{V}$ on $R^{4}$ leads to:

$$
\nabla .\left(\dot{x_{1}}, \dot{x_{2}}, \dot{x_{3}}, \dot{x}_{4}\right)^{T}=\frac{\partial f_{1}}{\partial x_{1}}+\frac{\partial f_{2}}{\partial x_{2}}+\frac{\partial f_{3}}{\partial x_{3}}+\frac{\partial f_{4}}{\partial x_{4}}=-(\rho+1)=f
$$

Note that $f=-(\rho+1)=-11$, for all positive values of $\rho$ that greater than zero, (1) is dissipative system. The exponential rate is:

$$
\frac{d V}{d t}=f V \Rightarrow V(t)=V_{0} e^{f t}=V_{0} e^{-11 t}
$$

The volume element $V_{0}$ from above equation is contracted by the flow into $V_{0} e^{-11 t}$ at time t.

\subsection{Graphical and numerical analysis}

The fourth and fifth order Runge-Kutta method is used to solve system (1). With initial values $\left.x\right|_{x_{1(0)}, x_{2(0)}, x_{3(0)}, x_{4(0)}}=[4,1,4,2]$.

\subsubsection{Wave form of new system (1)}

The wave-form $x_{1}(t), x_{2}(t), x_{3}(t)$ and $x_{4}(t)$ for system (1) is characterized with aperiodic structure, shown in Figure 1, which is the basic feature of chaotic system. 


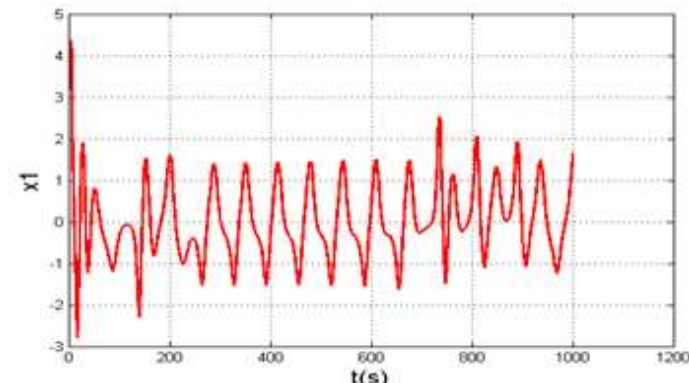

(a)

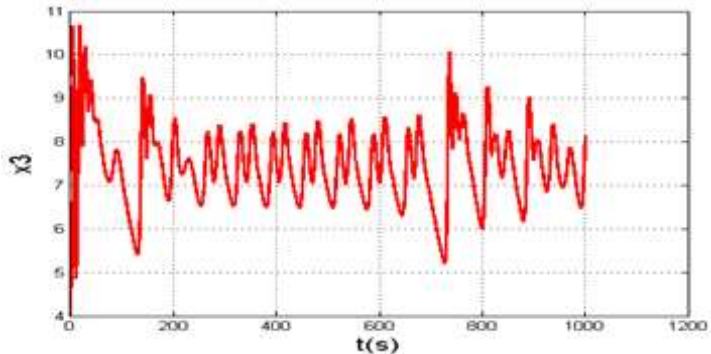

(c)

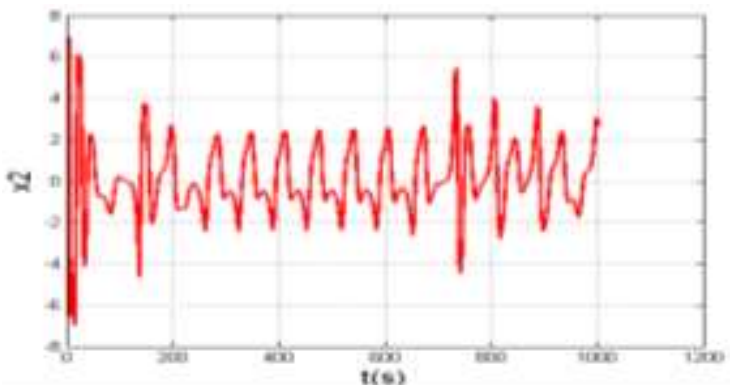

(b)

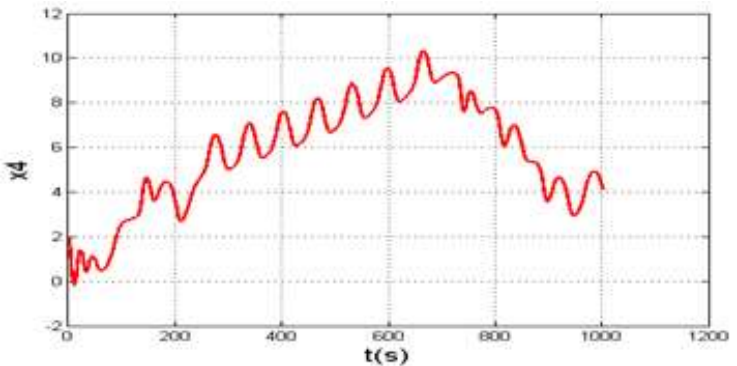

(d)

Figure 1. The wafe form of new system (1), (a): $x_{1}$ versus time; (b): $x_{2}$ versus time; (c): $x_{3}$ versus time; (d): $x_{4}$ versus time

\subsubsection{Phase portrait of the system (1)}

In this paragraph, Figure 2 and Figure 3 shows chaotic strange attractor for new system (1) in $\left(x_{1}, x_{2}, x_{4}\right)$ space, and chaotic strange attractor for new system $(1)$ in $\left(x_{1}, x_{4}\right)$ plane.

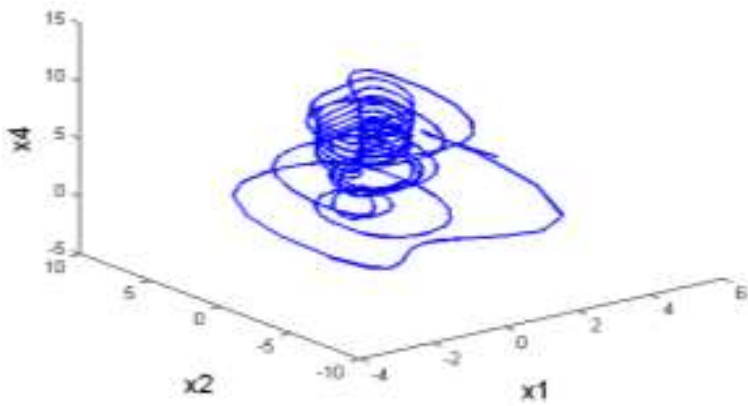

Figure 2. New system attractor in $\left(x_{1}, x_{2}, x_{4}\right)$

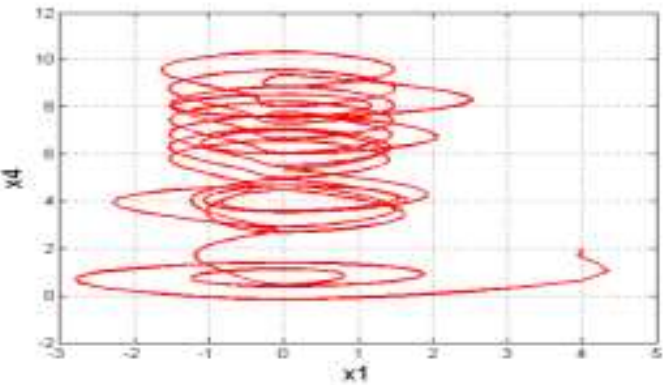

Figure 3. New system attractor in $\left(x_{1}, x_{4}\right)$

The new system exhibit chaotic attractor since the orbit appears dense in each graph.

\subsection{Lapiynuov exponent and lapiynuov dimension}

Generally the Lapiynuov exponent refers to average exponential rates of near trajectories that divergence in phase space. The new system said to be chaotic if there exist one positive Lapiynuov exponent at least. The values of lapiynuov exponents are: $\left(L_{1}=1.660748, L_{2}=0.149599, L_{3}=-0.068474\right.$ and $L_{4}=$ -12.144118). Therefore, the Lapiynuov dimension "Kaplan-Yorke dimension" of this system is:

$$
D_{L}=3+\frac{L_{1}+L_{2}+L_{3}}{\left|L_{4}\right|}=3.143433471
$$

Figure 4 show that the new system (1) is Highly Chaotic. 


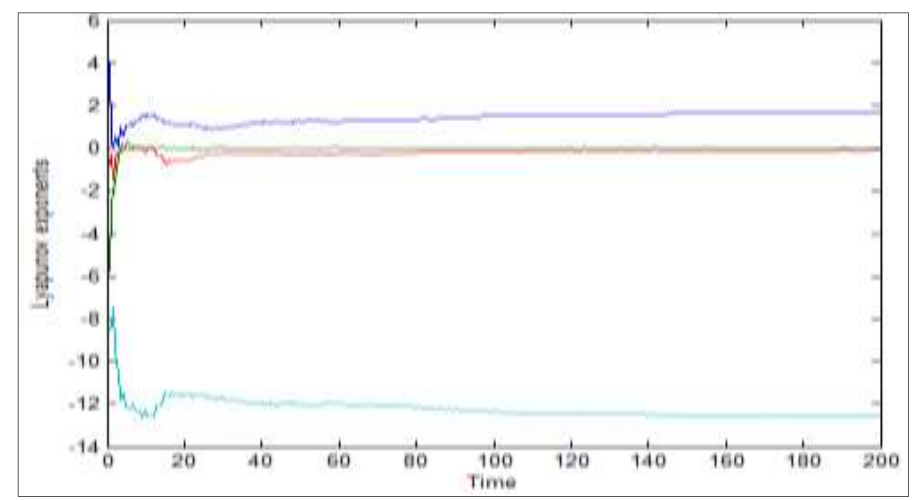

Figure 4. Lapiynuov exponent $\left(L_{1}, L_{2}, L_{3}, L_{4}\right)$ of new system (1)

\section{ADAPTIVE CONTROLLER DESIGN}

\subsection{Results theoretically}

An adaptive control strategy is design to stabilize highly chaotic system (1) with parameter $a$ which is unknown as follows

$$
\begin{aligned}
& \dot{x}_{1}=10\left(x_{2}-x_{1}\right)+u_{1} \\
& \dot{x}_{2}=a x_{1}-40 x_{1} x_{3}+x_{4}+u_{2} \\
& \dot{x}_{3}=10 x_{1} x_{2}-x_{3}+u_{3} \\
& \dot{x}_{4}=-8 x_{1}+u_{4} \\
& \text { where }\left[u_{1}, u_{2}, u_{3}, u_{4}\right]^{T} \text { are feedback controllers. }
\end{aligned}
$$

Now, we consider the subsequent adaptive control laws to make sure that the controlled system (5) converges asymptotically to the origin.

$$
\begin{aligned}
& u_{1}=-10\left(x_{2}-x_{1}\right)-\mu_{1} x_{1} \\
& u_{2}=-\hat{a} x_{1}+40 x_{1} x_{3}-x_{4}-\mu_{2} x_{2} \\
& u_{3}=-10 x_{1} x_{2}+x_{3}-\mu_{3} x_{3} \\
& u_{4}=8 x_{1}-\mu_{4} x_{4}
\end{aligned}
$$

Where $\mu_{1}, \mu_{2}, \mu_{3}$ and $\mu_{4}$ are constants, $\hat{a}$ is estimater of the parameter $a$.

Substituting the controller (6) into (5), we get

$$
\begin{aligned}
& \dot{x}_{1}=-\mu_{1} x_{1} \\
& \dot{x}_{2}=(\mathrm{a}-\hat{a}) x_{1}-\mu_{2} x_{2} \\
& \dot{x}_{3}=-\mu_{3} x_{3} \\
& \dot{x}_{4}=-\mu_{4} x_{4}
\end{aligned}
$$

Let the error of estimating parameter is

$$
e_{a}=a-\hat{a}
$$

Using (8), system (7) can be written as

$$
\begin{aligned}
& \dot{x}_{1}=-\mu_{1} x_{1} \\
& \dot{x}_{2}=e_{a} x_{1}-\mu_{2} x_{2} \\
& \dot{x}_{3}=-\mu_{3} x_{3} \\
& \dot{x}_{4}=-\mu_{4} x_{4}
\end{aligned}
$$

The Lapiynuov approach to deriving the update law is used to modify the parameter estimate $\hat{a}$. The quadratic lapiynuov function is considered as:

$$
V\left(x_{1}, x_{2}, x_{3}, x_{4}\right)=\frac{1}{2}\left(x_{1}{ }^{2}+x_{2}{ }^{2}+x_{3}{ }^{2}+x_{4}{ }^{2}+e_{a}{ }^{2}\right)
$$


which is a positive-definite on $R^{5}$.

$$
\text { Also } \quad \dot{e}_{a}=-\dot{\hat{a}}
$$

Differentiate $V \&$ substituting (9) and (11), we get:

$$
\dot{V}=-\mu_{1} x_{1}{ }^{2}-\mu_{2} x_{2}{ }^{2}-\mu_{3} x_{3}{ }^{2}-\mu_{4} x_{4}{ }^{2}-e_{a}\left[\dot{\hat{a}}-x_{1} x_{2}\right]
$$

Assume that

$$
\dot{\hat{a}}=x_{1} x_{2}+\mu_{5} e_{a}
$$

where $\mu_{5}$ is greater than zero.

Substitute (13) into (12), we get

$$
\dot{V}=-\mu_{1} x_{1}{ }^{2}-\mu_{2} x_{2}{ }^{2}-\mu_{3} x_{3}{ }^{2}-\mu_{4} x_{4}{ }^{2}-\mu_{5} e_{a}^{2}
$$

which is negative-definite on $R^{5}$

So, by Lapiynuov stability, Eigenvalues and Routh array criterion we get the result described below.

Proposition1. By adaptive control (6), where $\dot{\hat{a}}=x_{1} x_{2}+\mu_{5} e_{a}$ and $\mu_{1}, \mu_{2}, \mu_{3}, \mu_{4}$ and $\mu_{5}$ are positive constant, The chaotic system (5) is stabilized for $x(0) \in R^{4}$.

\subsection{Simulation and numerical results}

Simulation for controlled highly chaotic system (7) was done with $\left.x\right|_{x_{1(0)}, x_{2(0)}, x_{3(0)}, x_{4(0)}}=[-4,5,2,1]$ and $\left[\mu_{1}, \mu_{2}, \mu_{3}, \mu_{4}\right]=[30,40,50,10]$. Controlled state trajectories of new system (1), shown in Figure 5 .

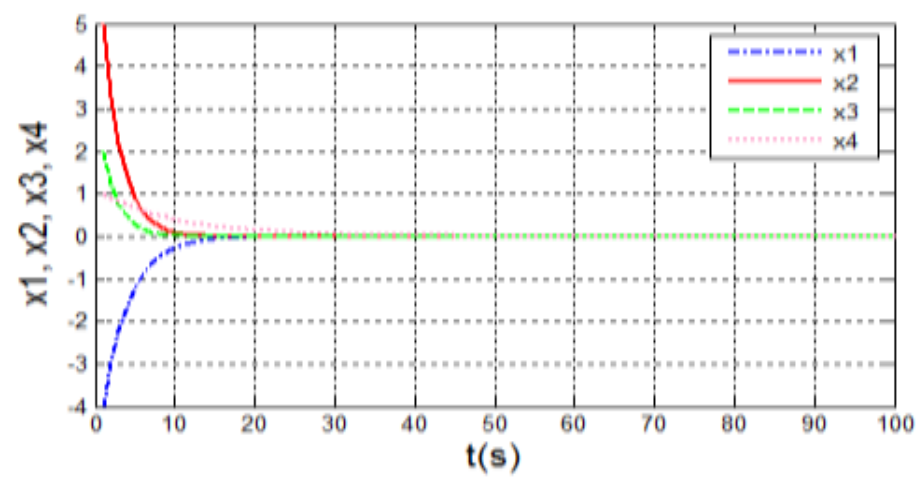

Figure 5. The behavior of state variables $x_{1}, x_{2}, x_{3}, x_{4}$ for the controlled system (7)

\section{A COMPARISON TABLES BEFORE AND AFTER CONTROL}

A comparison of eigenvalues shown in Table 2 and Routh array criteria shown in Table 3 of new system (1) before and after control at equilibrium point $(0,0,0,0)$.

Table 2. Eigenvalues of new system (1)

\begin{tabular}{ccc}
\hline Equilibrium point & Before Control & After Control \\
\hline \multirow{3}{*}{$(0,0,0,0)$} & $\lambda_{1}=-1 \quad \lambda_{2}=$ & $\lambda_{1}=-40$ \\
& -59.693 & $\lambda_{2}=-30$ \\
& $\lambda_{3}=0.026984$ & $\lambda_{3}=-50$ \\
& $\lambda_{4}=49.666$ & $\lambda_{3}=-10$ \\
\hline
\end{tabular}


Table 3. Calculated values of Routh array criteria of new system (1)

\begin{tabular}{cccccccc}
\hline Equilibrium point & $\lambda$ & \multicolumn{3}{c}{ Before Control } & \multicolumn{4}{c}{ After Control } \\
\hline & $\lambda^{4}$ & 1 & -2955 & 80 & 1 & 4820 & 60000 \\
$(0,0,0,0)$ & $\lambda^{3}$ & 11 & -2885 & 0 & 130 & 36800 & 0 \\
& $\lambda^{2}$ & -2692.727 & 80 & 0 & 4536.923 & 60000 & 0 \\
& $\lambda^{1}$ & -2884.67 & 0 & 0 & 35080.77 & 0 & 0 \\
& $\lambda^{0}$ & 80 & 0 & 0 & 60000 & 0 & 0 \\
\hline
\end{tabular}

\section{ADAPTIVE SYNCHRONIZATION STRATEGY}

\subsection{Theoretical results}

In this section we explain Adaptive synchronization strategy of highly chaotic system when the parameter $a$ is unknown.

As a drive system, we consider the highly chaotic dynamics represented by

$$
\begin{aligned}
& \dot{x}_{1}=10\left(x_{2}-x_{1}\right) \\
& \dot{x}_{2}=a x_{1}-40 x_{1} x_{3}+x_{4} \\
& \dot{x}_{3}=10 x_{1} x_{2}-x_{3} \\
& \dot{x}_{4}=-8 x_{1}
\end{aligned}
$$

where $a$ is the unknown system parameter .

For response system, we consider the controlled dynamics represented as

$$
\begin{aligned}
& \dot{y}_{1}=10\left(y_{2}-y_{1}\right)+u_{1} \\
& \dot{y}_{2}=a y_{1}-40 y_{1} y_{3}+y_{4}+u_{2} \\
& \dot{y}_{3}=10 y_{1} y_{2}-y_{3}+u_{3} \\
& \dot{y}_{4}=-8 y_{1}+u_{4}
\end{aligned}
$$

Where $u_{1}, u_{2}, u_{3}, u_{4}$ are the designed non-linear controllers and $y_{i},(i=1,2,3,4)$ are the state variables. Adaptive Synchronization error given by

$$
e_{i}=y_{i}-x_{i},(i=1,2,3,4)
$$

Hence, the error dynamics:

$$
\begin{aligned}
& \dot{e}_{1}=10\left(e_{2}-e_{1}\right)+u_{1} \\
& \dot{e}_{2}=a e_{1}-40\left(e_{1} e_{3}+x_{3} e_{1}+x_{1} e_{3}\right)+e_{4}+u_{2} \\
& \dot{e}_{3}=10\left(e_{1} e_{2}+x_{2} e_{1}+x_{1} e_{2}\right)-e_{3}+u_{3} \\
& \dot{e}_{4}=-8 e_{1}+u_{4}
\end{aligned}
$$

define adaptive control functions $u_{1}(t), u_{2}(t), u_{3}(t)$ and $u_{4}(t)$ as:

$$
\begin{aligned}
& u_{1}=-10\left(e_{2}-e_{1}\right)-\mu_{1} e_{1} \\
& u_{2}=-\hat{a} e_{1}+40\left(e_{1} e_{3}+x_{3} e_{1}+x_{1} e_{3}\right)-e_{4}-\mu_{2} e_{2} \\
& u_{3}=-10\left(e_{1} e_{2}+x_{2} e_{1}+x_{1} e_{2}\right)+e_{3}-\mu_{3} e_{3} \\
& u_{4}=8 e_{1}-\mu_{4} e_{4}
\end{aligned}
$$

where $\mu_{1}, \mu_{2}, \mu_{3}, \mu_{4}$ are positive constant, and $\hat{a}$ is the estimater of parameter $a$. Substituting (19) into (18), we get the dynamic of synchronization error as:

$$
\begin{aligned}
& \dot{e}_{1}=-\mu_{1} e_{1} \\
& \dot{e}_{2}=(a-\hat{a}) e_{1}-\mu_{2} e_{2} \\
& \dot{e}_{3}=-\mu_{3} e_{3} \\
& \dot{e}_{4}=-\mu_{4} e_{4}
\end{aligned}
$$

Now, the error of the parameter estimater is:

$$
e_{a}=a-\hat{a}
$$

Substitute (21) into (20), we get 


$$
\begin{aligned}
& \dot{e}_{1}=-\mu_{1} e_{1} \\
& \dot{e}_{2}=e_{a} e_{1}-\mu_{2} e_{2} \\
& \dot{e}_{3}=-\mu_{3} e_{3} \\
& \dot{e}_{4}=-\mu_{4} e_{4}
\end{aligned}
$$

From the Lapiynuov approach to deriving the update law is used to modify the parameter estimate. The quadratic lapiynuov function is considered as:

$$
V=\frac{1}{2}\left(e_{1}^{2}+e_{2}^{2}+e_{3}^{2}+e_{4}^{2}+e_{a}^{2}\right)
$$

which is positive definite on $R^{5}$.

$$
\text { Note that } \quad \dot{e}_{a}=-\dot{\hat{a}}
$$

Differentiating $V$ on the trajectories of (22) and use (24), we obtain

$$
\dot{V}=-\mu_{1} e_{1}^{2}-\mu_{2} e_{2}^{2}-\mu_{3} e_{3}^{2}-\mu_{4} e_{4}^{2}-e_{a}\left[\dot{\hat{a}}-e_{1} e_{2}\right]
$$

In (25), the estimated parameter is updated by:

$$
\dot{\hat{a}}=e_{1} e_{2}+\mu_{5} e_{a}
$$

where the constant $\mu_{5}$ is a positive.

Substituting (24) into (23), we get

$$
\dot{V}=-\mu_{1} e_{1}^{2}-\mu_{2} e_{2}^{2}-\mu_{3} e_{3}^{2}-\mu_{4} e_{4}^{2}-\mu_{5} e_{a}^{2}
$$

Which is a negative on $R^{5}$.

Hence, by Lapiynuov stability [22], immediately the error of synchronization and the error of parameter estimate degeneration exponentially to zero. Thus, the following is proved.

Proposition2. The identical chaotic systems, the drive (15) and the response (16) with unknown parameter $a$ are synchronized for each initial value by adaptive control technique (19), where the estimated parameter given by (26) and the constant $\mu_{i},(i=1,2,3,4,5)$ are possitive.

\subsection{Numerical simulation and results}

Runge-Kutta method of order fourth was used to solve (15) \& (16), and also solve the dynamic of synchronization error (20). The initial values of the drive system_(15) as $\left.x\right|_{x_{1(0)}, x_{2(0)}, x_{3(0)}, x_{4(0)}}=[2,15,10,3]$ and the response system (16) as $\left.y\right|_{y_{1(0)}, y_{2(0)}, y_{3(0)}, y_{4(0)}}=[18,6,4,3]$, parameter value as $a=296.5$ and $k_{i}=4$ for $i=1,2,3,4$. Adaptive synchronization of chaotic system shown in Figure 6, and convergent for system (20) shown in Figure 7.

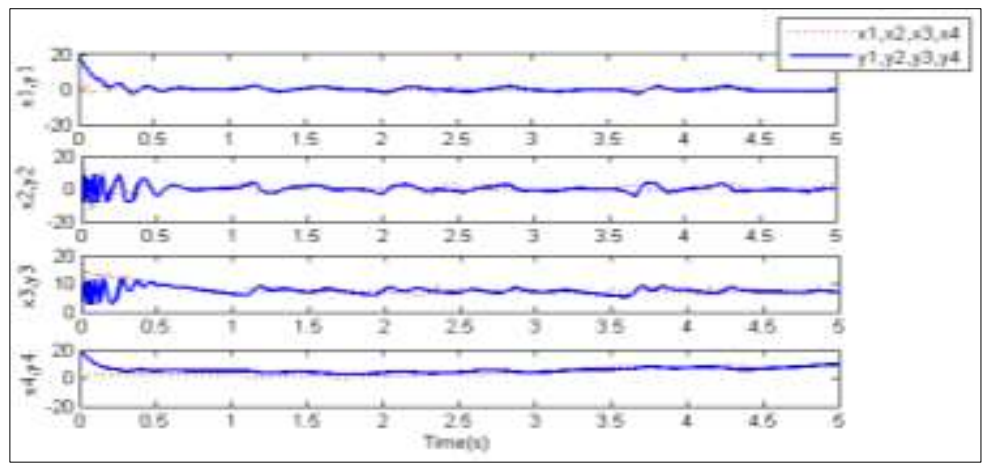

Figure 6. Trajectories by adaptive synchronization for drive system (15) and response system (16) 


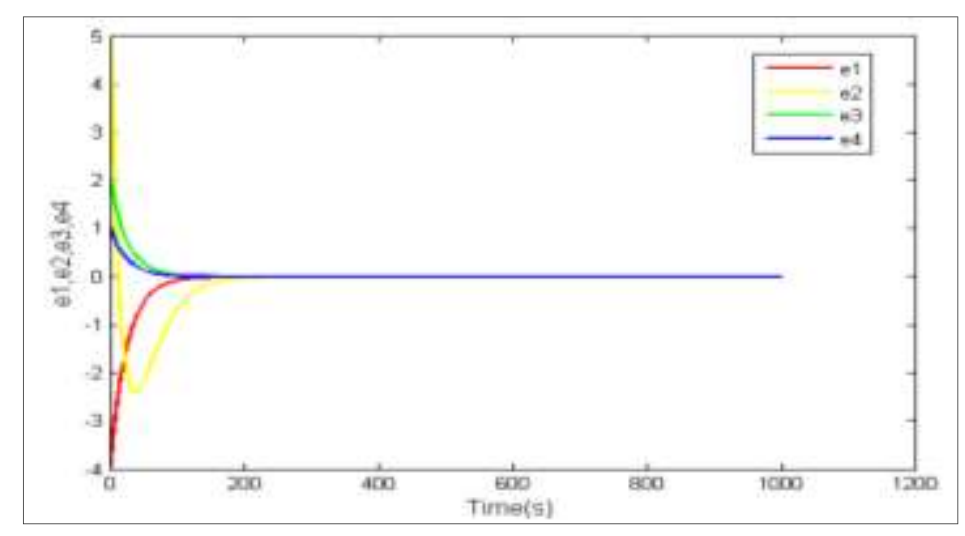

Figure 7. Convergence of trajectories for the dynamic of synchronization error (20)

\section{CONCLUSION}

This paper presents a consideration of a new four-dimension on continuous-time system with quadratic cross-product nonlinear terms, its solution through the fourth and fifth order Runge-Kutta method. The characteristics of the system analyzed by mean of equilibrium points, analysis of stability (such as Lapiynuov function, Routh criterion and characteristic equation roots) all these methods shows that the new system is unstable. The analysis of dissipativity shows that the new dynamical system is dissipative for all values of the parameters $\mathrm{a}, \delta, \varphi, \mathrm{k}$ and for the positive values of parameter $\rho$. Lapiynuov exponent, lapiynuov dimension "Kaplan-Yorke dimension" and analysis of wave-form presence the chaotic behaviors when the parameters taken as $\rho=10, \mathrm{a}=296.5, \delta=40, \varphi=10, \mathrm{k}=8$, and the maximum values of lapiynuov exponents for the system are $L_{1}=1.660748, L_{2}==0.149599, L_{3}==-0.068474$ and $L_{4}==-12.144118$, Lapiynuov dimension "Kaplan-Yorke dimension" of new system is $D_{L}=2.22349544$, that means the new system is highly chaotic. Furthermore, by adaptive control strategy we stabilized the highly chaotic system. Finally, adaptive synchronization was established for identical highly chaotic system with unknown parameter, so we got the synchronization error convergence to zero. Moreover; we compared graphical and theoretical results of the new system before and after control.

\section{ACKNOWLEDGEMENTS}

We would like sincerely thank and also acknowledge to the university of Mosul and college of computer science and mathematics for the support and encouragement that help us to improve the quality of this work.

\section{REFERENCES}

[1] Maysoon M. Aziz and M. A. Hamid, "The possibility of Increasing the Predictability Indices After Control of 3D Continuous-Time system," International conference on computing and Information Science and Technology and their Application, 2019.

[2] S. F. Al-Azzawi, et al., Stability of Lorenz System at the Second Equilibria Point based on Gardano's Method. Journal of Physics: Conference Series, vol.1477, 022009, 2020.

[3] S. F. Al-Azzawi and Maysoon M.Aziz, "Strategies of Linear Feedback Control and Its classification", TELKOMNIKA Telecommunication Computing Electronics and Control, vol.17, no.4, pp.1931-1940, 2019.

[4] Maysoon M. Aziz and S. F. AL-Azzawi, "Hybrid Chaos Synchronization Between Two Different Hyperchaotic Systems via Two Approaches," Optik, vol.138, pp. 328-340, 2017.

[5] Z. Sh. Al-Talib and S. F. AL-Azzawi, "Projective and Hybrid Projective Synchronization of 4-D Hyperchaotic System via Nonlinear Controller Strategy," TELKOMNIKA Telecommunication, Computing, Electronics and Control, vol. 18, no 2, pp. 1012-1020, April 2020.

[6] B. Jovic, "Synchronization Techniques for Chaotic Communication Systems", Springer-Verlag, Berlin Heidelberg, 2011.

[7] Maysoon.M.Aziz and Mansour.N. Faraj, "Numerical and Chaotic Analysis of Chua's Circuit," Journal of Emerging trends in computing and information science, vol.3, no.5,pp. 783-791, 2012.

[8] S. F. Al-Azzawi, et al., "Chaotic Lorenz System and it's Suppressed," Journal of Advanced Research in Dynamical and Control Systems, vol.12, no. 2, pp. 548-555, 2020.

[9] S. F. Al-Azzawi and Maysoon M..Aziz, "Chaos Synchronization of Nonlinear Dynamical Systems via a Novel Analytical Approach,” Alexandria Engineering Journal, vol. 57, no. 4, pp. 3493-3500, Dec 2018. 
[10] G. Chen and T. Ueta, "Yet another chaotic attracor," Int. J. Bifurcation Chaos, vol.9, pp 1465- 1466,1999.

[11] F. Yu and C. Wang, "Generation of a New Three-Dimensional Autonomous Chaotic Attractor and Its Four Wing Type," Engineering Technology and Applied Science Research, vol.3, no.1, pp 352- 358, 2013.

[12] V. Sundarapandian and I. Pehlivan "Analysis, Control, Synchronization and Circuit Design of a Novel Chaotic System," Maths. Comp. Mod., vol. 55, pp 1904-1915, 2012.

[13] A.S. Pikovsky et al., "Onset of Stochasticity in Decay Confinement of Parametric Instability", Sov. Phys. JETP, vol.47, pp.715-719, 1978

[14] S. Y. Al-hayali and S. F. AL-Azzawi, "An Optimal Nonlinear Control For Anti-Synchronization of Rabinovich Hyperchaotic System," Indonesian Journal of Electrical Engineering and Computer Science, vol. 19, no. 1, pp 379386, July 2020.

[15] S. Y. Al-hayali and S. F. AL-Azzawi, "An Optimal Control For Complete Synchronization of 4D Rabinovich Hyperchaotic Systems," TELKOMNIKA Telecommunication, Computing, Electronics and Control, vol. 18, no 2, pp. 994-1000, Apr 2020.

[16] A. S. Al-Obeidi and S. F. Al-Azzawi "Chaos Synchronization in a 6-D Hyperchaotic System with Self-Excited Attractor", TELKOMNIKA Telecommunication Computing Electronics and Control, vol.18, no.3, pp. 1483-1490, June 2020.

[17] Z. Sh. Al-Talib and S. F. AL-Azzawi, "Projective Synchronization for 4D Hyperchaotic System Based on Adaptive Nonlinear Control Strategy," Indonesian Journal of Electrical Engineering and Computer Science, vol. 19, no. 2, pp. 715-722, Aug 2020.

[18] K.M. Cuomo et al., "Synchronization of Lorenz-based chaotic circuits and application to secure communication", IEEE Transaction on Circuits and Systems-II:Analogue and Digital Processing, vol. 40, no.10, pp. 626-633, 1993.

[19] M. Gh. Saeed et al.,"Multiple Models of Binary-Support-Vector-Machine for Face Verification Using Histogram Orientation Gradient Features" Journal of Theoretical and Applied Information technology, vol.96, no.19, pp.63496360, October 2018.

[20] F. L. Malallah et al.,"Off-Line Arabic (Indian) Numbers Recognition Using Expert System," International Journal of Advanced computer science and Applications, vol.7, no. 4, pp.397-406, 2016.

[21] Maysoon.M Aziz, "Stability analysis of mathematical model," International Journal of science and research (IJSR), Vol.7, no. 9, pp. 147-148, Sep. 2018.

[22] W.Hahn, "The Stability of Motion", Springer, New York,1967.

[23] Maysson M.Aziz and S. F. Al-Azzawi, “Anti-synchronization of Nonlinear Dynamical Systems Based on Gardano's Method," Optik, vol. 134, pp. 109-120, Apr 2017.

[24] Maysoon M.Aziz and Z. A. Al-Nuaimi,"stability and hop-Bifurcation for Diabetes Model", International journal of electronics communication and computer Engineering, vol.4, no.3, pp. 805-809, 2013.

[25] J. Joseph and A. Ivan, "Feedback and Control Systems". McGraw-Hill, 1990. 